\title{
Optical response of nanostructured metal/dielectric composites and multilayers
}

\author{
G.B. Smith*, A.I. Maaroof, R.S. Allan, S. Schelm, G.R. Anstis and M.B. Cortie \\ Applied Physics and Institute of Nanoscale Technology, University of Technology, Sydney, PO Box \\ 123 Broadway NSW 2007 Australia g.smith@uts.edu.au
}

\begin{abstract}
The homogeneous optical response in conducting nanostructured layers, and in insulating layers containing dense arrays of self assembled conducting nanoparticles separated by organic linkers, is examined experimentally through their effective complex indices $\left(\mathrm{n}^{*}, \mathrm{k}^{*}\right)$. Classical effective medium models, modified to account for the 3-phase nanostructure, are shown to explain $\left(\mathrm{n}^{*}, \mathrm{k}^{*}\right)$ in dense particulate systems but not inhomogeneous layers with macroscopic conductance for which a different approach to homogenisation is discussed. $\left(\mathrm{n}^{*}, \mathrm{k}^{*}\right)$ data on thin granular metal films, thin mesoporous gold, and on thin metal layers containing ordered arrays of voids, is linked to properties of the surface plasmon states which span the nanostructured film. Coupling between evanescent waves at either surface counterbalanced by electron scattering losses must be considered. Virtual bound states for resonant photons result, with the associated transit delay leading to a large rise in $n^{*}$ in many nanostructures. Overcoating $\mathrm{n}-\mathrm{Ag}$ with alumina is shown to alter $\left(n^{*}, k^{*}\right)$ through its impact on the SP coupling. In contrast to classical optical homogenisation, effective indices depend on film thickness. Supporting high resolution SEM images are presented.
\end{abstract}

Keywords: nanostructured metal films, effective medium, surface plasmons, porous metal

\section{INTRODUCTION : TOPOLOGY AND EFFECTIVE MEDIUM APPROACHES}

Far field optical data such as reflectance, transmittance, or ellipsometry parameters, for thin metal films containing nanoscale voids or nanostructured surfaces, allows a representation of such films as homogenised effective mediums if they cause weak or negligible scattering. That is for a plane incident wave the far field transmitted and reflected radiation are also uniform plane waves. It is also important to note that the effective medium layer is assumed to be smooth. The linking of this observed homogeneous optical behaviour to inhomogeneous composition and structure, is called effective medium or optical homogenisation theory ${ }^{1,2}$. Classical effective medium models deal with nanostructures with feature size relative to wavelength $\lambda$ typically less than $\lambda / 10$. Many examples exist where these theories work well for metal particle arrays where there is no macroscopic conduction. Successful examples of these include $\mathrm{Cr} / \mathrm{CrO}_{3}$ and $\mathrm{Mo} / \mathrm{WO}_{3}$ cermet layers for solar selective absorbers ${ }^{3,4}$, anisotropic columnar cermet layers with oriented rod like nanoparticle metal for angular selectivity ${ }^{5,6}$, and metal nanoparticles or metal islands on ceramic substrates ${ }^{7}$. Separated metal particles embedded in another medium, can often be handled by Maxwell Garnett ${ }^{8,9}$ type models when multipoles on each particle do not arise. A sphere then has a single wavelength, three fold degenerate surface plasmon resonance, which lifts in a spheroid to two or three distinct resonant modes. In very dilute arrays with no shape variability, resonances are at wavelengths satisfying $\varepsilon_{m}^{\prime}=-\varepsilon_{h}(1 / L-1)$ where $\mathrm{L}$ is one depolarisation factor, $\varepsilon_{m}^{\prime}$ is the real part of the metal's dielectric constant and $\varepsilon_{h}$ is the dielectric constant of the insulating host medium. It is usually considered that at high particle densities multipoles and hence multiple resonances, must also occur. However random symmetry in the array can cancel higher order poles at quite high density provided particle separation is maintained, as we will demonstrate experimentally with a particular class of cermets. These are spherical gold nanoparticles self assembled in solution using organic linkers prior to deposition ${ }^{10}$. This system is the represented in Figure 1(a). Figure 1 shows a range of topologies, which are introduced for dense metal containing layers so as to link different effective medium approaches to different nanostructures, and in particular to distinguish those in which surface plasmon phenomena will play a role. Figure $1(\mathrm{a}-\mathrm{d})$ cover situations where classical theories are applicable.

Correspondence to g.smith@uts.edu.au 


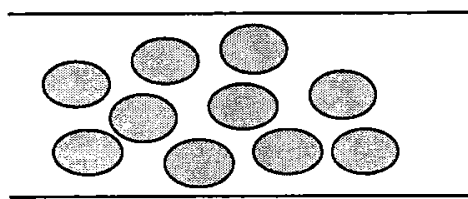

(a) Dense random array metal nanoparticles

(Maxwell-Garnett)

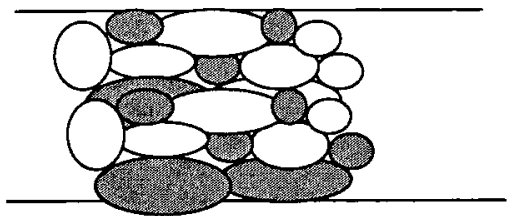

(b) Non -conducting (c) Conducting (both Bruggemann)

Two phase granular system
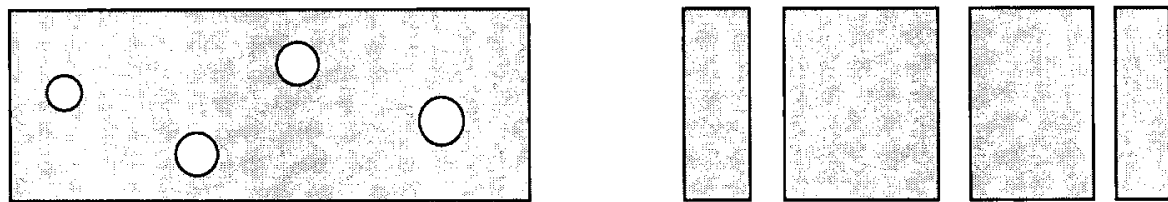

Cross section NanoVoids in thin metal layers

(d) No Surface plasmons (Maxwell Garnett)

(e) Surface plasmons both sides

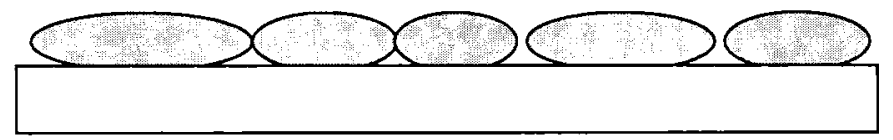

(f) Nano-Granular metal film cross section - surface plasmon mechanisms both sides - nanostructured surface and penetrating voids

Figure 1 Various thin film metal topologies and associated effective medium models

Once metal particles or metal islands join up sufficiently to allow macroscopic conductance two main topological possibilities arise; either the insulating medium present, which may be void or a dielectric, forms isolated regions, or it also percolates. The former mix (void in metal) was assumed to give the inverse type of Maxwell Garnett situation as in Fig. 1(d). A Bruggemann type model might apply if either Fig. 1(b) topologies arise where there is still no macroscopic conduction or if both percolate as in 1(c). These cases may occur in the transition zone where concentrations are about equal. One or more plasmon resonance wavelengths arise in all of these models. These are usually linked to surface plasmons (SP) which can be isolated on a particle or void as in 1(a) and 1(d), or at one or both surfaces of the film. It is the latter that is our main concern and can occur in the topologies in Figures 1(c) and 1 (d), and from now on we label these as boundary surface plasmons BSPs (also surface plasmon polaritons) to distinguish them from the local SPs on isolated particles. Three void situations are shown which must be distinguished. Fig.1(d) has internal voids but a smooth surface so boundary surface plasmons cannot be formed. Figures 1(e) and 1(f) show cases where BSPs can be generated by incoming radiation. In 1(e) the array of voids allows surface momentum to be acquired and in $1(f)$ two sources of surface momentum contribute to generation of BSPs (i) a nanostructured film surface due to the grain or particle profile (ii) arrays of transverse voids that cross the layer. In some cases just the nanostructured surfaces may occur. In our thin granular metal samples the observed topology (see section 2) allows both effects.

Thus the classical models in many experiments will not describe far field data once macroscopic conductance occurs, even at low void concentrations where they might be expected to apply. Other special situations with macroscopic 
conductance and distinct responses include chains of touching particles as found in some gold blacks ${ }^{11}$. Touching and intersecting plasmonic particles have a distinct resonant feature. They have a continuum of resonant wavelengths ${ }^{12}$, which experimentaily shows up as a broad absorption band extending to long wavelengths.

The experimental results we present show that the classical models are inappropriate when macroscopic conductance occurs in a metal nanostructure at frequencies below the plasma frequency. This is because the effective complex refractive indices $\left(n^{*}, k^{*}\right)$ must then account for the existence of boundary surface plasmons (BSPs) on their outer surfaces. In traditional thin metal films with smooth surfaces, even if they contain internal voids, this is not an issue because surface plasmons cannot form. However if a thin metal layer has surface nanostructure on either surface, with voids that penetrate through the film a special case, coupled BSPs can form. Their ability to influence film transmittance thus depends primarily on whether they form on both surfaces ${ }^{13}$, and on film thickness. The forming of a coupled SP state is well known in thin films ${ }^{13-16}$ but until recently has not played a large role in thin film optics. It is also known to occur in plasma layers ${ }^{16}$. Non-radiative losses within the metal, and surface radiative losses, normally limit the influence of BSP coupling, but situations can arise where it can be have a major impact. Recent interest in anomalous transmittance in metal films with penetrating voids is an example ${ }^{14,15,18}$. In principle even very weak coupling between two oscillators can lead to complete energy transfer from one to the other. A well known example is close planar optical wave guides where evanescent waves from one guide penetrate the other. Damping is the factor limiting energy transfer in practice. In our case energy at the illuminated surface transfers to the exit surface, also via coupling of evanescent waves. In a lossless metal layer $100 \%$ transmittance would thus occur for some nanostructures and the Poynting vector calculation associated with our simulations (section 4) clearly shows the energy channels through the voids. We will show an interesting variation of $\mathrm{n}^{*}$ as structure varies and the balance between reflectance and transmittance alters, when $\mathrm{k}^{*}=0$. Results for the same ordered structures for real metals will be compared to the ideal metal to show the influence of damping on BSP coupling and hence effective indices.

$\left(\mathrm{n}^{*}, \mathrm{k}^{*}\right)$ data on metal layers with different nanostructures; mesoporous gold, granular silver films and ordered arrays of voids in silver, will be compared to the ideal case. All but ordered arrays of voids in silver are random nanostructures but common features between ordered and random void arrays are found. The most common feature seen will be the elevation of $n^{*}$, which is discussed in terms of the resonant nature of the coupled SP state for incident photons, in particular the resonant delay. Some simple relations for experimentally determining the SP dispersion relations in terms of $n^{*}, k^{*}$ are examined. Finally the effective optical response, especially $n^{*}$, is shown to be quite sensitive to the dielectric properties of neighbouring layers, as is to be expected when BSP's are dominating optical response. This is done experimentally using an oxide coating on a granular metal film.

\section{SAMPLES AND NANOSTRUCTURE}

Micrographs of the three experimental nanoporous metal systems studied here appear in figure 2 . They are respectively (i) dense self-assembled gold in a dithiol matrix (ii) granular silver thin film (iii) microporous gold. In addition for comparisons and fundamental understanding of some key issues, simulations and exact models have been done for regular arrays of slits across a metal layer of varying thickness. A brief description of preparation and structure follows.

\subsection{Dense but insulating metal arrays}

Gold nanoparticles $6 \mathrm{~nm}$ in diameter are self assembled for a short period in a solution containing alkane dithiol molecules which form bridges between particles ${ }^{10,19}$. Resultant aggregates are deposited onto nanoporous alumina substrates. The film thickness is determined by the solution volume filtered and the spacing between gold nanoparticles. A key feature in these films is seen in figure 2(a), namely the phase separation of the voids in the final film. The other key issue is the particle randomness. The most dense array we have produced has linkers with only 2 carbons (C2dithiol) between sulfur groups, so spacing between particles is a few $\mathrm{nm}$. Hence the gold occupies $74 \%$ of the volume in the linker-gold system. The disorder in the gold array has a significant impact on the homogenised optical response.

\subsection{Granular silver thin films.}

Noble metal films in which the quantity of metal deposited is above the amount needed to give macroscopic conductivity but not enough to give complete coverage of the substrate are well known [18] and occur for most vacuum deposition processes, but at different deposited mass according to energetics of the incoming species. The samples 
discussed are single layer thin films of $\mathrm{n}$ - $\mathrm{Ag}$ and double layer $\mathrm{Al}_{2} \mathrm{O}_{3} / \mathrm{n}$ - $\mathrm{Ag}$ deposited using high-pressure dc-magnetron sputtering. An example is in Figure 2(b). As thickness increases from the onset of percolation $(5$ to $6 \mathrm{~nm}$

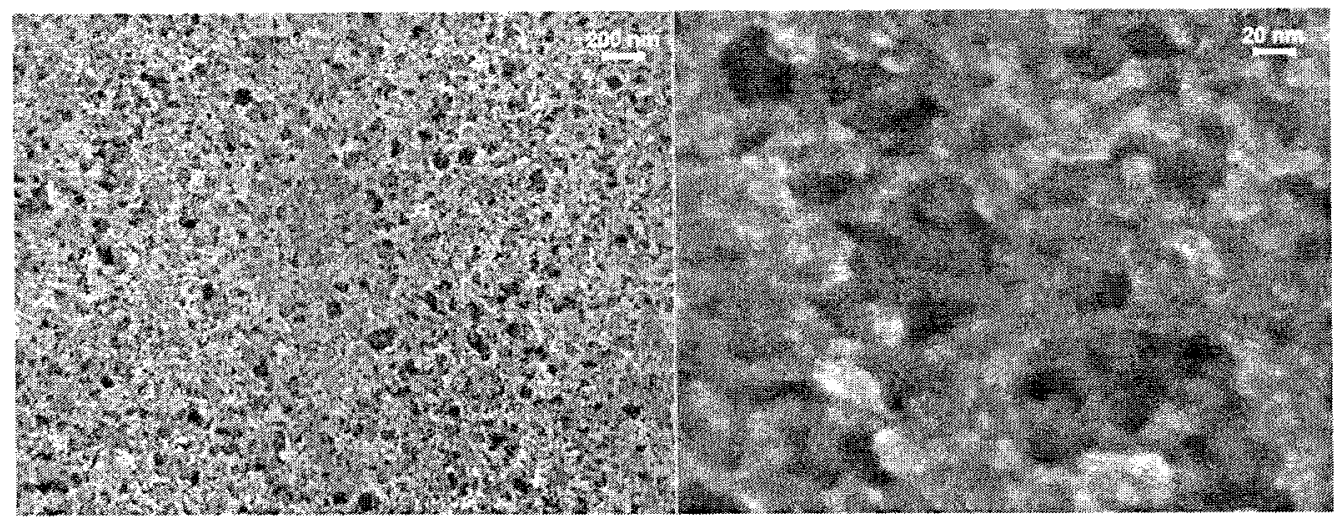

Figure 2(a) High resolution FEGSEM images of self assembled gold nanoparticle films embedded in linkers. A mesoporous void network makes up the third phase in these images.

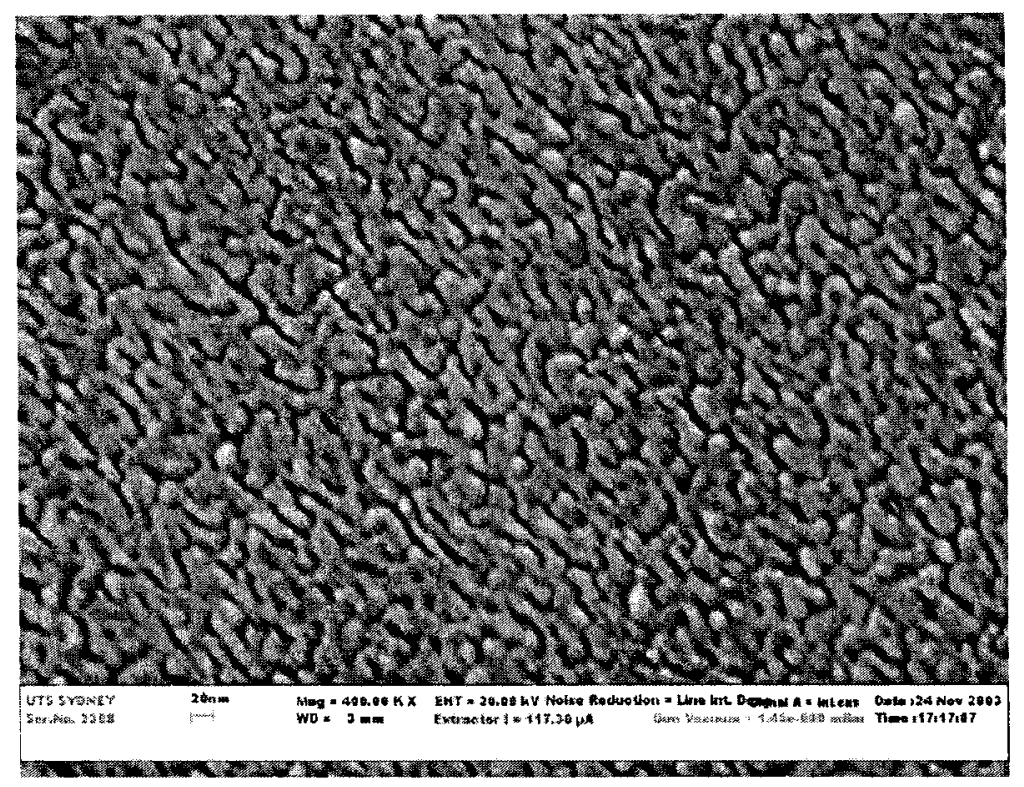

Figure 2(b) High resolution FEGSEM images of a granular silver film $7 \mathrm{~nm}$ thick with elongated nanovoids.

here), void density decreases and distinct grain boundaries show up between nano-grains. Residual voids are still present at 10 to $11 \mathrm{~nm}$. As long as they occur we find anomalous optical response. The aluminium oxide overcoat is used to examine the impact of an overcoat on effective optical indices. $7 \mathrm{~nm}$ and $10 \mathrm{~nm}$ thick silver films are characterised.

\subsection{Mesoporous gold.}

Co-deposits of gold and aluminium were evaporated to $180 \mathrm{~nm}$ thickness onto a $10 \mathrm{~nm}$ layer of chromium on a glass slide. The relative amounts of each element were aimed at producing $\mathrm{AuAl}_{2}$. The aluminium was then etched out using a sodium hydroxide solution. This left a mesoporous gold network as shown in figure 2(c). As can be seen in the micrograph the void volume fraction is substantial. Both gold and void phases form complex networks in this 
mesoporous system, so it has a distinct morphology compared to the other systems in this study. Isolated voids are also present.

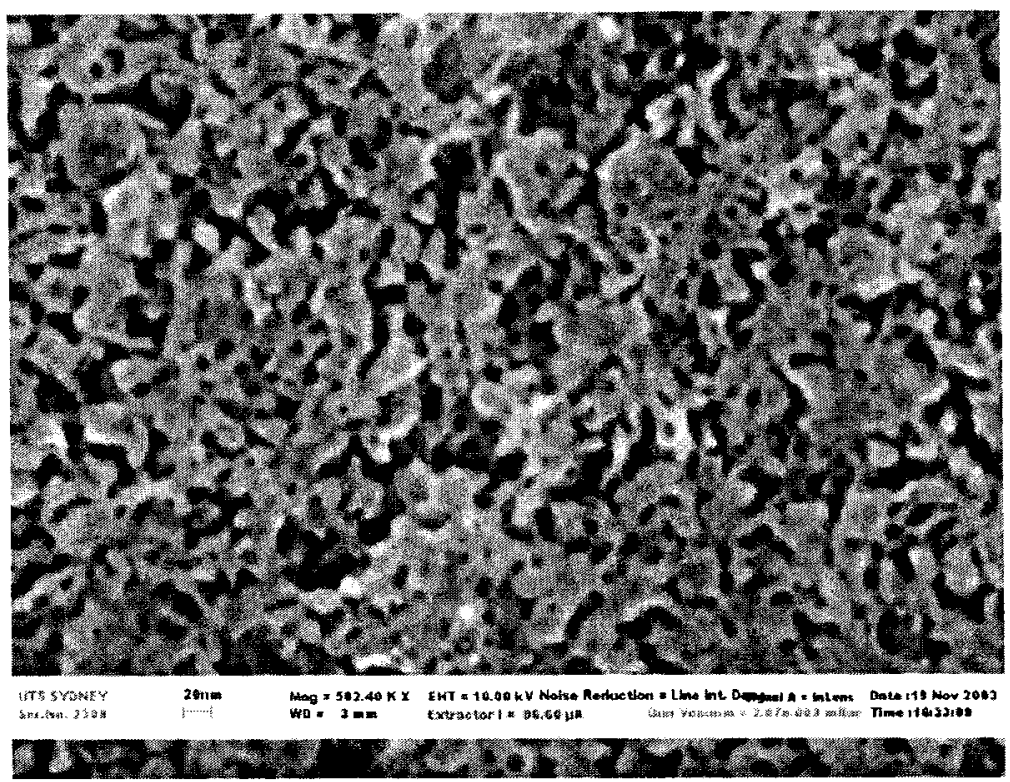

Figure 2(c) High resolution FEGSEM images of a mesoporous gold film.

2.3 Ordered arrays of slits in thin metal (computer simulation studies).

An array of slits $200 \mathrm{~nm}$ wide with centres spaced at $400 \mathrm{~nm}$ intervals spanning metal layers of thickness from very thin to a micron, were studied. Two types of metal were considered (i) a perfect lossless metal for which informative analytic models of optical response follow (ii) silver.

\section{SOME MODELS}

3.1 Double effective medium model

This model is fully described in reference 19 . Gold nanospheres dispersed in a medium of dense dithiol linker can be represented as an effective medium using the well known Maxwell Garnett expression for spheres in a dielectric medium. This gold-linker medium has effective dielectric constant $\varepsilon_{\mathrm{AuL}}$. The complete medium is then represented as a new composite medium made up of spheres of the medium described by $\varepsilon_{\mathrm{AuL}}$ admixed with voids. In most cases the situation in figure 1(b) is appropriate in this final step so the Bruggemann expression of equation (1) gives the complete mediums optical response in terms of the quantity $\varepsilon^{*}=\left(n^{*}+j k^{*}\right)^{2}$ and $f_{\text {voids }}$ is the volume fraction of voids.

$\left(1-f_{v o i d s}\right) \frac{\varepsilon_{A u L}-\varepsilon^{*}}{\varepsilon_{A u L}+2 \varepsilon^{*}}+f_{v o i d s} \frac{1-\varepsilon^{*}}{1+2 \varepsilon^{*}}=0$

\subsection{Surface plasmons and homogenised optical constants}

The $n^{*}, k^{*}$ data on granular silver thin films and mesoporous gold films that follows is interpreted in terms of surface plasmons on both sides of the layer coupling together. The homogenised model assumes a standard smooth surface thin film situation and for simplicity in discussion here we take the symmetric case with the same oxide and the same surface 
structure on both sides of the nanostructured metal layer. It is straightforward to generalise. In considering reflectance and transmittance and also resonant effects it is useful to work in terms of surface admittance $Z=E_{x} / H_{y}$ in each medium at each interface, where the normal to the surface is along the $\mathrm{z}$-axis. From Maxwell's equations the simple metal has $Z$ $=\mathrm{Z}_{\mathrm{m}}=\left(\mathrm{k}_{\mathrm{z}} / \mathrm{k}_{\mathrm{o}}\right) / \varepsilon_{\mathrm{m}}$ with $\varepsilon_{\mathrm{n}}$ being the dielectric constant of the metal and $\mathrm{k}_{\mathrm{o}}=2 \pi / \lambda$, the wavevector in free space.

Surface impedances of interest are that for the smooth effective medium layer $Z_{m}{ }^{*}, Z_{o x}$ the smooth oxide either side of the effective medium, $Z_{o x}{ }^{S P}$, the oxide layer above and below the actual nanostructured surface supporting surface plasmons and $Z_{\mathrm{m}}{ }^{\mathrm{SP}}$, the nanostructured metal layer with surface plasmons. These are defined in terms of $\varepsilon_{\mathrm{ox}}$ and $\boldsymbol{\varepsilon}_{m}$ the dielectric constant of the oxide and metal respectively. $\mathrm{k}_{\mathrm{x}}$ is the wavector parallel to the surface, and $\mathrm{n}^{*}, \mathrm{k}^{*}$ the effective indices. $\mathrm{k}_{\mathrm{x}}$ exists for normal incidence only when SPs are present. Each $\mathrm{Z}$ becomes,

$$
\begin{aligned}
& Z_{o x}=\frac{k_{z}^{o x} / k_{o}}{\varepsilon_{o x}}=\frac{1}{\sqrt{\varepsilon_{0 x}}}=\frac{1}{n_{o x}} \\
& Z_{m}^{*}=\frac{k_{z m}^{*} / k_{o}}{\varepsilon_{m}^{*}}=\frac{1}{\sqrt{\varepsilon_{m}^{*}}}=\frac{1}{n^{*}+j k^{*}} \\
& Z_{o x}^{S P}=\frac{k_{z o x}^{S P} / k_{o}}{\varepsilon_{o x}}=\frac{\sqrt{\varepsilon_{o x}-\left(k_{x} / k_{o}\right)^{2}}}{\varepsilon_{o x}}=Z_{o x} \sqrt{1-\left(\Delta_{S P} / \varepsilon_{o x}\right)} \\
& Z_{m}^{S P}=\frac{k_{z m}^{S P} / k_{o}}{\varepsilon_{m}}=\frac{\sqrt{\varepsilon_{m}-\left(k_{x} / k_{o}\right)^{2}}}{\varepsilon_{m}}=Z_{m} \sqrt{1-\left(\Delta_{s P} / \varepsilon_{m}\right)}
\end{aligned}
$$

In equation (5) the case of a metal layer with a nanostructured surface is given. With penetrating voids different impedances $Z_{\text {medium/SP }}$ would be needed to describe the void channels to replace $Z_{m}$. However the structure in these equations is preserved. $\Delta_{\mathrm{SP}}$ represents the effect of the surface momentum on impedance and it and hence $\mathrm{k}_{\mathrm{x}}$ can be derived at each wavelength from $n^{*}, k^{*}$ data using these relations. One can go further and use these surface impedances together with film structure to study resonance issues such as delay times which are linked primarily to $\mathrm{n}^{*}$. The link between $n^{*}, k^{*}$ and $k_{x}$ can be derived by equating transmittance or reflectance for the structure in terms of both models

For the symmetric oxide/metal/oxide case the amplitude reflection ratio $r$ for a layer of thickness $\mathrm{d}$ becomes

$$
r=\frac{r_{o m}^{*}+r_{m o}^{*} e^{j 2 k_{z}^{*} d}}{1+r_{o m}^{*} r_{m o}^{*} e^{j 2 k_{z}^{*} d}}=\frac{r_{o m}^{S P}+r_{m o}^{S P} e^{j 2 k_{z m} d}}{1+r_{o m}^{S P} r_{m o}^{S P} e^{j 2 k_{z m} d}}
$$

with the first form applicable to the smooth effective medium structure and the second to the nanostructure supporting surface plasmons. Each $r$ is expressed in terms of surface impedances as

$$
r_{o m}^{*}=\frac{Z_{o x}-Z_{m}^{*}}{Z_{o x}+Z_{m}^{*}} \quad \text { and } \quad r_{o m}^{S P}=\frac{Z_{o x}^{S P}-Z_{m}^{S P}}{Z_{o x}^{S P}+Z_{m}^{S P}}
$$


Note that within the layer the wavevectors in each version of equation (6) for normal incidence are $k_{z}^{*}=k_{o}\left(n^{*}+j k^{*}\right)$ within the effective medium and $\boldsymbol{k}_{z m}=\boldsymbol{k}_{o}(\boldsymbol{n}+\boldsymbol{j} \boldsymbol{k})$ with $(\mathrm{n}, \mathrm{k})$ metal indices for the layer with a nanostructured surface. $\mathrm{k}_{\mathrm{zm}}$ changes when voids cross the layer. The measured reflectance is $R=|r|^{2}$.

These equations are readily modified for related systems and for different angles of incidence. A formal effective medium solution based on nanostructure and composition requires first principles establishment of the SP dispersion relations for $\mathrm{k}_{\mathrm{x}}$ and knowledge of internal impedances for each structure. However with far field experimental data we can, using equations (2 -7), establish the dispersion relations and impedances experimentally. This also enables us to learn a lot about related matters such as resonant delay times without direct measurement. Note that from equation (6) the effective medium models must take account of surface plasmon modified electromagnetic behaviour (through $Z_{\mathrm{ox}}{ }^{\mathrm{SP}}$ ) in the neighbouring media as well as internally. It is thus obvious from equations (6) and (7) that $\mathrm{n}^{*}, \mathrm{k}^{*}$ depend on both layer thickness and the dielectric properties of the neighbouring layers. One is led to wonder just how much published data over the years on optical constants of thin film metal layers has been influenced by these surface effects. Unless the data is for surfaces known to be quite smooth then data presented will be an effective medium result. Using graded indices to allow for non-smooth metal surfaces at the nanoscale (a common practice over many years which continues today) does not incorporate the essential physics.

It is possible to restructure these equations to model the input admittance or impedance of the layer and substrate oxide combined. At resonance this $Z_{\text {input }}$ will be close to the value of outer layer ( $Z_{0 x}$ in the above models) and then low reflectance and enhanced transmittance and/or absorptance become possible. If the $Z$ 's match perfectly $R=0$. If the coating is a lossless medium the system must be lossless to match so then $\mathrm{T}=100 \%$. The slit array system outlined in section 2.3 is useful for gaining insights into such resonances and for a perfect lossless metal it predicts at a fixed wavelength, a set of thicknesses at which $\mathrm{T}=100 \%$, as shown in figure 3 for $750 \mathrm{~nm}$ radiation. Also shown is the result for the same structure in silver to demonstrate the impact of damping in real metals. The third plot is a simple single mode analytic model for the perfect metal ${ }^{14}$. Much enhanced transmittance is retained for these sub -wavelength

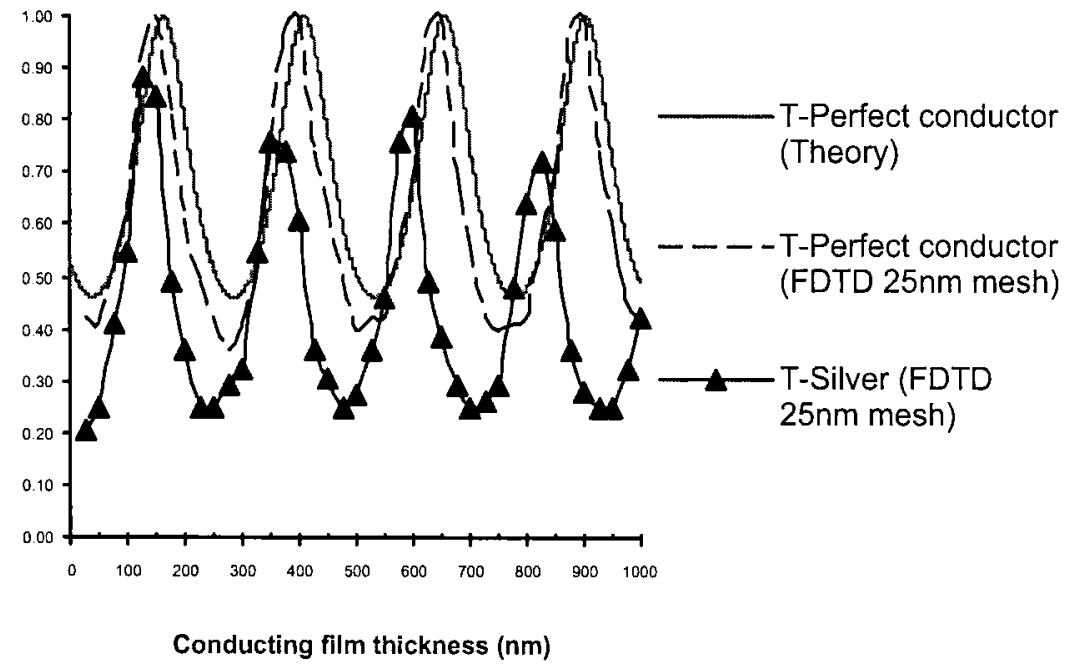

Figure 3. Periodic behaviour of transmittance with thickness in an ideal metal layer and silver at $750 \mathrm{~nm}$, slit width $200 \mathrm{~nm}$, slit spacing $400 \mathrm{~nm}$. 
apertures in real metals, as a result of coupling of BSPs via these apertures. The input admittance is real for the ideal metal, and oscillates between $\mathrm{n}_{\mathrm{ox}}$ when $\mathrm{T}=100 \%, \mathrm{R}=0$ and a high value at maximum $\mathrm{R}$ of around $67 \%$. At maximum $\mathrm{R}, \mathrm{Z}_{\text {input }}$ for this structure is around $6.5 \mathrm{n}_{\mathrm{ox}}$. Resonant delay times are largest at resonance. This system can thus provide a perfect antireflection coating while the real metal array (silver) can transmit around $80 \%$ of the incident radiation at thickness as high as $700 \mathrm{~nm}$. A simple model for lossless metal with an ordered array of sub-wavelength slits spanning the layer, can be used, as here, to describe long wavelength properties when the structural scales are sub-wavelength ${ }^{14}$. It has been established by diffraction of the incoming wave with $k_{x}=0$ into the $T E_{10}$ waveguide mode with $k_{x}= \pm 2 \pi / w$. w is the slit array lattice parameter and all other modes are neglected. The evanescent wave in the oxide layers is localised near the interface and decays into a propagating reflected or transmitted waves. This simple model is increasingly divergent from exact simulations at wavelengths below $800 \mathrm{~nm}$, for the $200 \mathrm{~nm}$ slit width and slit centre separation of $400 \mathrm{~nm}$ that were used in the simulation runs in this report. That is for wavelengths under twice the slit separation this simplified model breaks down. In the full simulation studies there is also a qualitative shift in $T$ and $R$, and hence $n^{*}, k^{*}$ spectral character for wavelengths above and below $800 \mathrm{~nm}$ (see section 4). These qualitative shifts are similar to those very recently predicted for single metallic slits by Bravo-Adad et $\mathrm{al}^{21}$. They occur when wavelength changes cause characteristic scales to change from being sub-wavelength to larger than wavelength. This represents a shift to a geometric optics regime for the slits or the array and also the onset of a multimode theory as opposed to a single mode model. This long wavelength limit single mode model for slits in metal is useful, and is somewhat analogous to classical quasistatic effective medium approximations. Slit width a here, like particle size in classical effective medium models, must be much smaller than wavelength and lattice constant $L$ must also be reasonably large (like particle spacing) to avoid higher order modes. Outgoing waves with $\mathrm{k}_{\mathrm{x}}$ non zero will also start to show up once this single mode model breaks down, signalling that optical homogenisation is no longer physically relevant.

\section{OPTICAL PROPERTIES : EFFECTIVE REFRACTIVE INDICES}

Both deposited films and simulated layers give fully specular behaviour above $550 \mathrm{~nm}$, within the order of accuracy of measurements. Samples were checked with scattering apparatus and the simulations for the nature of the far field outgoing waves. This means we can use far field responses to extract effective optical constants. Reflectance and transmittance spectra across the visible and near infra red range are modelled using measured average thickness from cross section images, and standard smooth thin film equations. The modelling parameters are $\mathrm{n}^{*}, \mathrm{k}^{*}$ and are adjusted for best fit across the complete spectrum. Measured ellipsometric parameters were used to check results in some cases. Spectral $n^{*}, k^{*}$ data is presented in Figures 4 for measured samples (a) self assembled gold with C2 linkers, (b) and (c) 7 $\mathrm{nm}$ and $10 \mathrm{~nm}$ thick granular silver both bare and then with a $15 \mathrm{~nm}$ coating of $\mathrm{Al}_{2} \mathrm{O}_{3}$. The $10 \mathrm{~nm}$ thick film has voids similar to those in fig. 2(b) but less of them (c) $180 \mathrm{~nm}$ thick mesoporous gold.

The fit shown to the self assembled $6 \mathrm{~nm}$ diameter gold nanoparticle data is with a double classical effective medium model $^{19}$ and is shown in figure 4(a). It uses a Maxwell Garnett (MG) model for spheres embedded in linker and a Bruggemann model for this MG "medium" mixed with voids. With this preparation technique voids are phase separated. The void fraction is the only adjustable parameter used in fitting and best fit values are in good accord with images.

The simulated reflectance and transmittance results are also processed to extract $n^{*}, \mathrm{k}^{*}$ as for the measured data and appear in figure 5 for the perfect metal, and for silver in the same structure. The array of $200 \mathrm{~nm}$ wide slits is spaced with centres $400 \mathrm{~nm}$ apart, and each figure has a set of curves comparing silver with the ideal lossless metal. Thicknesses of the metal layer are varied. Fig. 5(a) compares the thickness dependence of $n^{*}$ and $k^{*}$ for the ideal metal and silver for $750 \mathrm{~nm}$ radiation. Fig. 5 (b) shows the spectral dependence of $\mathrm{n}^{*}, \mathrm{k}^{*}$ for a $50 \mathrm{~nm}$ thick layer and fig. 5(c) that for a $500 \mathrm{~m}$ thick layer. It is of interest to compare the $50 \mathrm{~nm}$ layer to the thin film data in Fig. 4(b). It is also interesting because the perfect metal has a quite different response to silver for these thinner layers, while they are much closer when both are much thicker. Small oscillations in simulated data are mainly due to errors associated with the FDTD mesh size used of $25 \mathrm{~nm}$. A finer mesh will smooth these but elongates computational time substantially. 


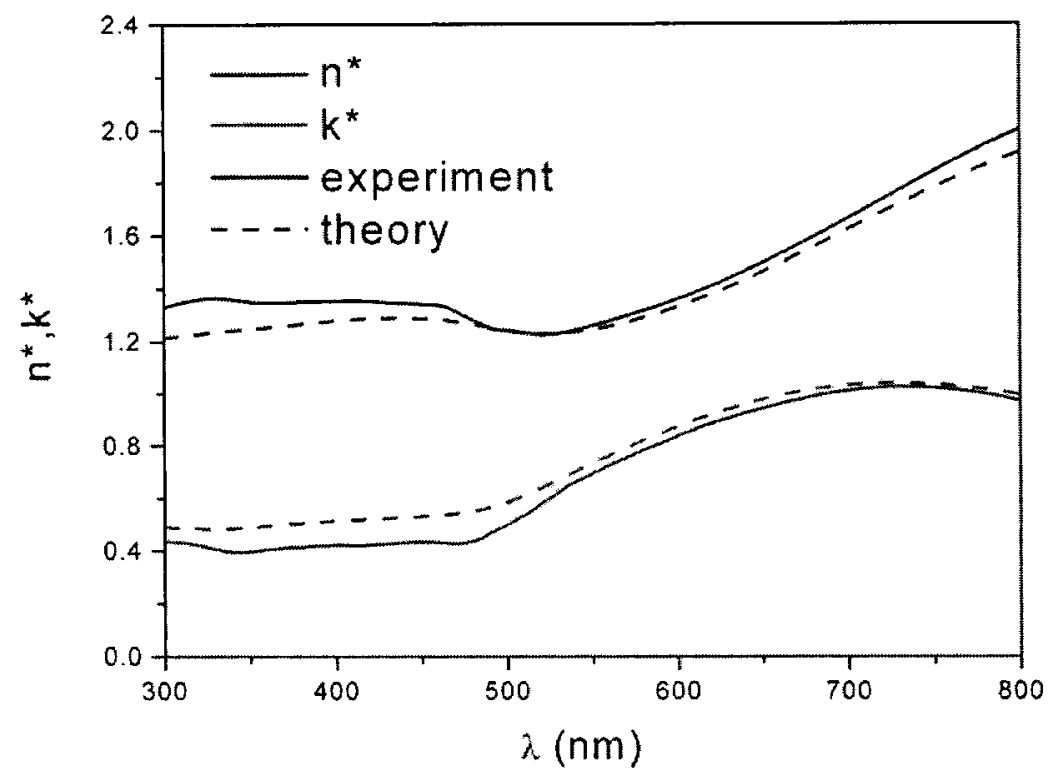

Figure 4(a) Effective indices $n^{*}, k^{*}$ for self assembled gold films along with results of fitting with a double effective medium model in which the only fitting parameter is the void fraction.

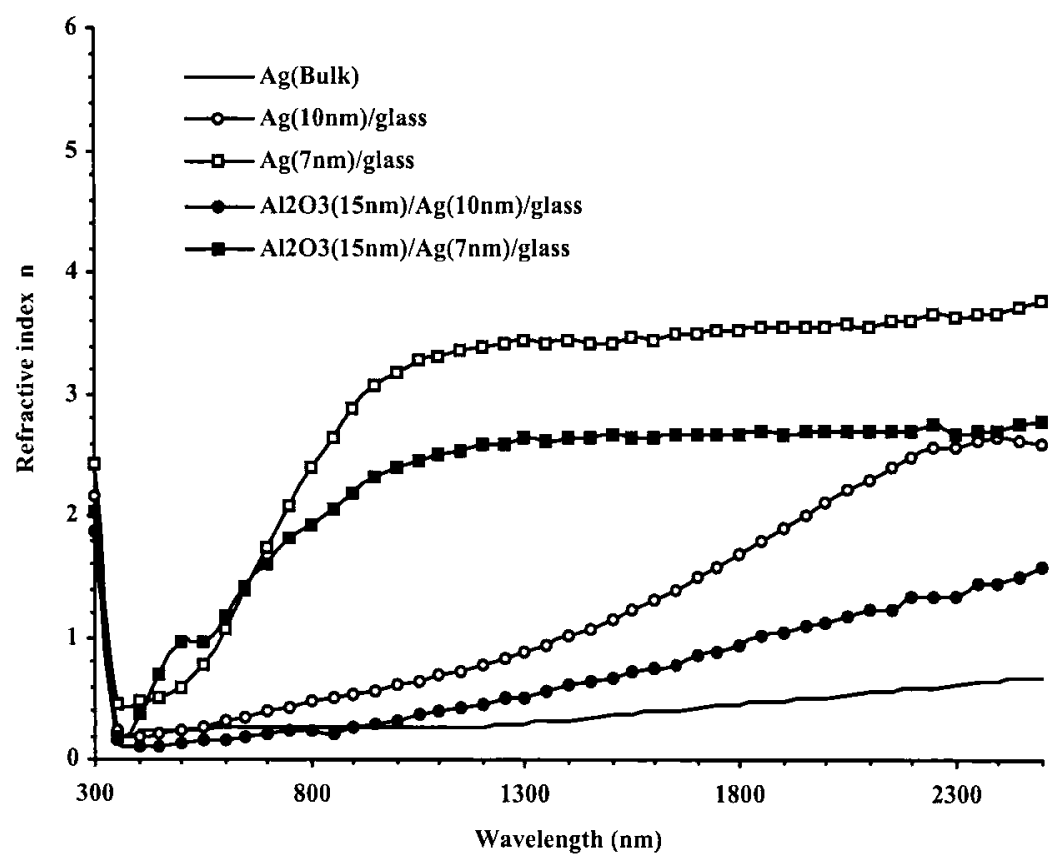

Figure 4(b) Effective $\mathrm{n}^{*}$ for $7 \mathrm{~nm}$ and $10 \mathrm{~nm}$ thick granular silver, both bare and with an aluminum oxide overcoating. 


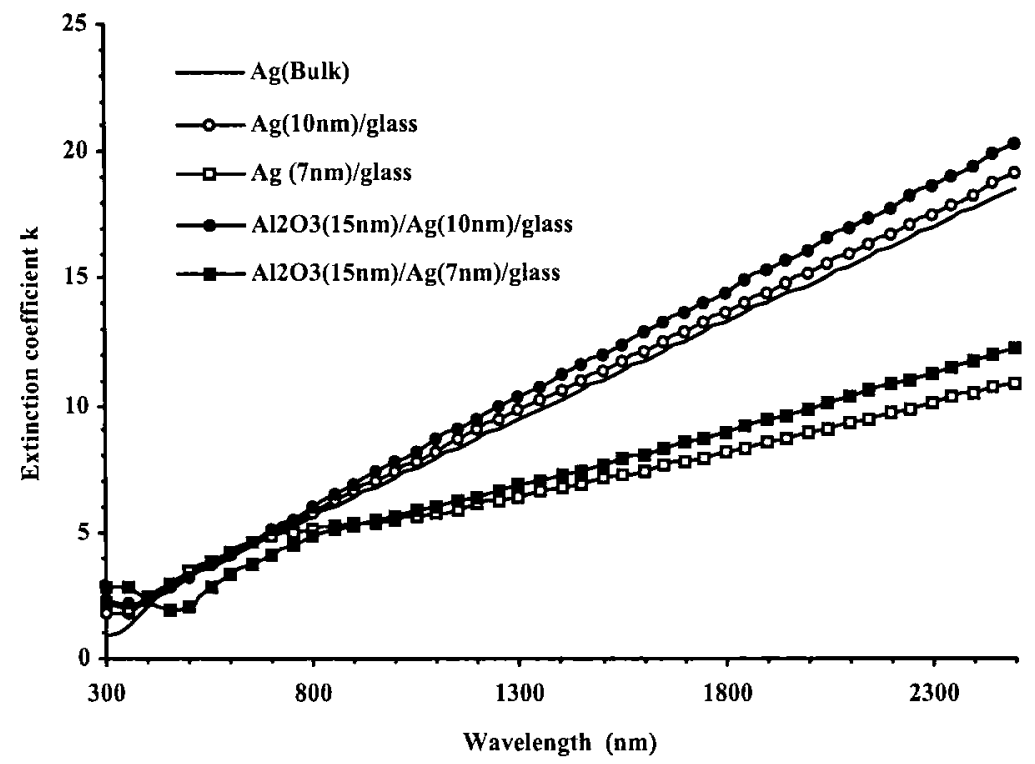

Figure $4(\mathrm{c})$ Effective $\mathrm{k}^{*}$ values for $7 \mathrm{~nm}$ and $10 \mathrm{~nm}$ thick granular silver films both bare and with aluminum oxide overcoatings.

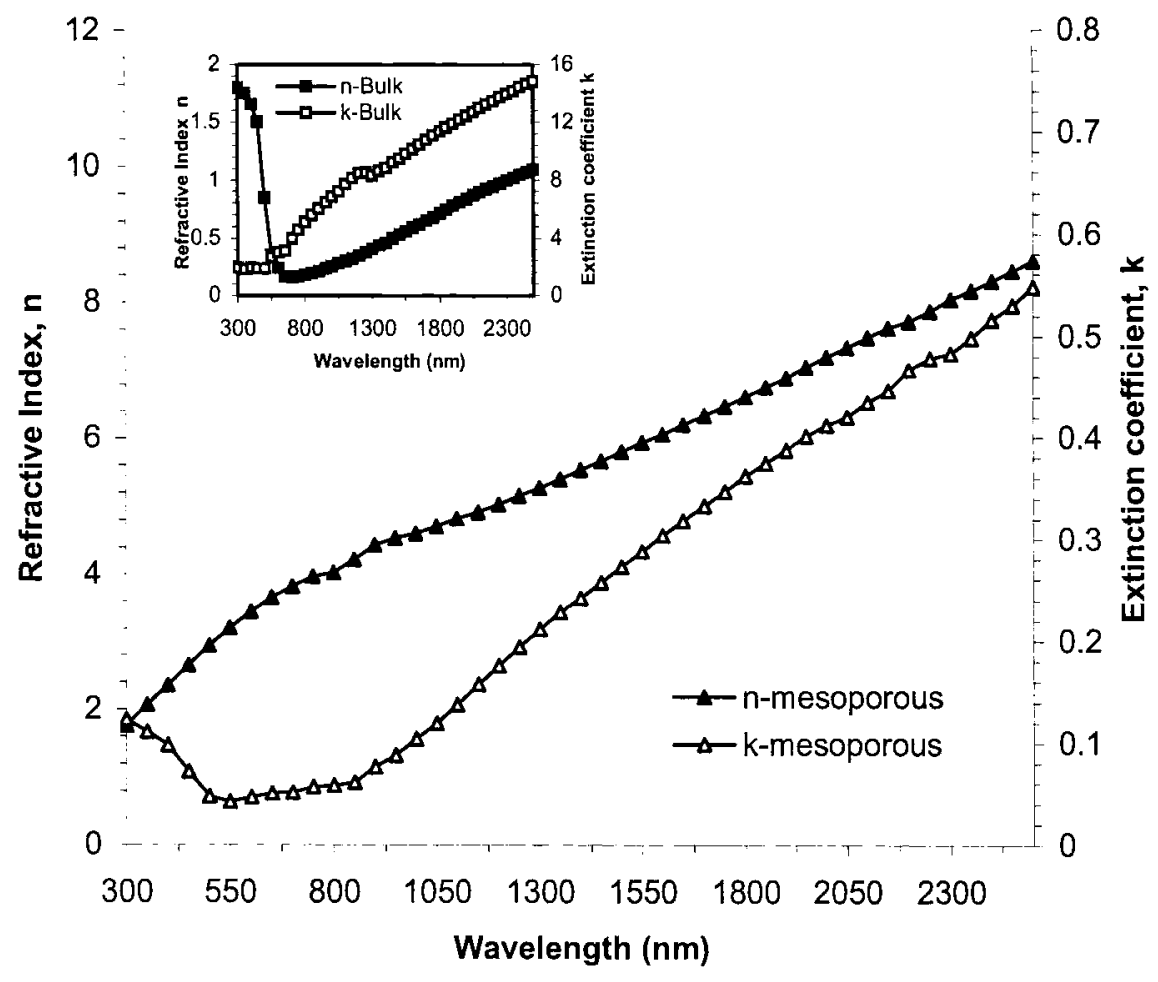

Figure 4(d) Effective $\mathrm{n}^{*}$ and $\mathrm{k}^{*}$ for an $180 \mathrm{~nm}$ thick mesoporous gold film. 


\section{DISCUSSION}

All results in figure 4 and 5, except for the "dense gold in linker mixed with voids" case, display responses which cannot be explained by classical quasi-static effective medium model situations as outlined in conjunction with Figure 1. An important feature about the self-assembled gold is the indications that even at $74 \%$ of gold in linker, MG models for the gold-linker phase still work well. This is due to the randomness of the gold particles in the arrays, despite their fixed separation, and was predicted by Smith some years $\mathrm{ago}^{22}$. We had to wait until 2003, and self-assembly techniques for reasonable experimental proof. In practice either aggregation and many touching particles normally occurs at such densities, or if particles are coated with dielectric to avoid such touching at high density ${ }^{9}$, the metal particles plus shell are in effect close-packed into semi-ordered arrays which then have multipolar quasistatic modes once metal fill factors are above about $40 \%$. All the other systems in Figures 4 and 5 support BSPs, which in turn affects optical homogenisation. Common qualitative features in $\mathrm{n}^{*}$ and $\mathrm{k}^{*}$ are apparent. The most striking is the significant enhancement of $n^{*}$ relative to $n$ in bulk silver or gold, in which $n$ is below 1 at most wavelengths of interest. The distinguishing features between the mesoporous gold network and the granular thin film system are twofold. Both have elevated $\mathrm{n}^{*}$ but the spectral dependence of $\mathrm{n}^{*}$ is much stronger in the mesoporous system. Their $\mathrm{k}^{*}$ are also different. The granular film shows a significant reduction in $\mathrm{k}^{*}$ from bulk silver but it is still strong, but in the mesoporous gold case $\mathrm{k}^{*}$ drops even further. It is interesting that $\mathrm{n}^{*}$ and $\mathrm{k}^{*}$ versus wavelength in the mesoporous system are roughly parallel to $\mathrm{n}$ and $\mathrm{k}$ in bulk gold but $\mathrm{n}$ is much enhanced and $\mathrm{k}$ much reduced.

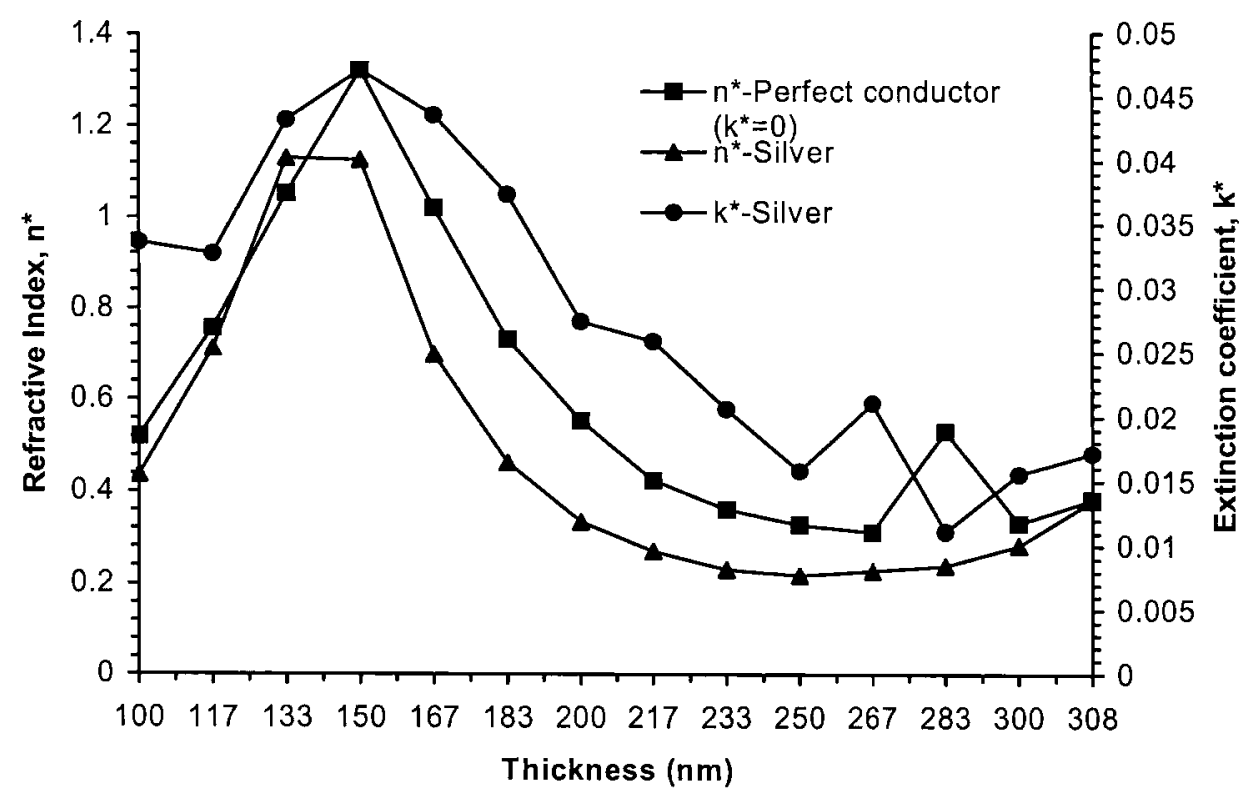

Figure 5(a) Effective $\mathrm{n}^{*}$ and $\mathrm{k}^{*}$ versus thickness for thin metal with an array of slits showing response of ideal metal and silver (conductor) with the same structure. 


\section{Results for $A g=500 \mathrm{~nm}$}

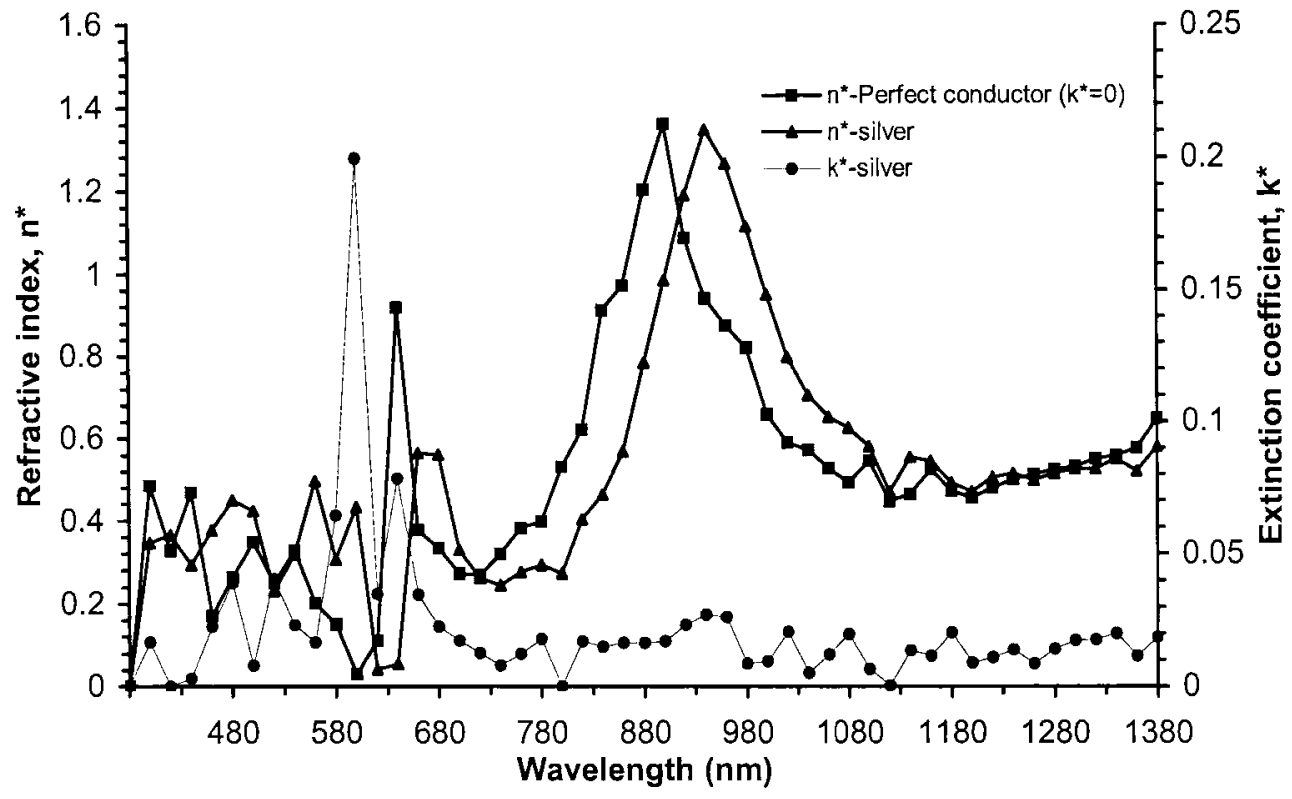

Figure 5 (b) Spectral $\mathrm{n}^{*}$ and $\mathrm{k}^{*}$ values for an array of slits in $500 \mathrm{~nm}$ thick layers of perfect conductor and silver

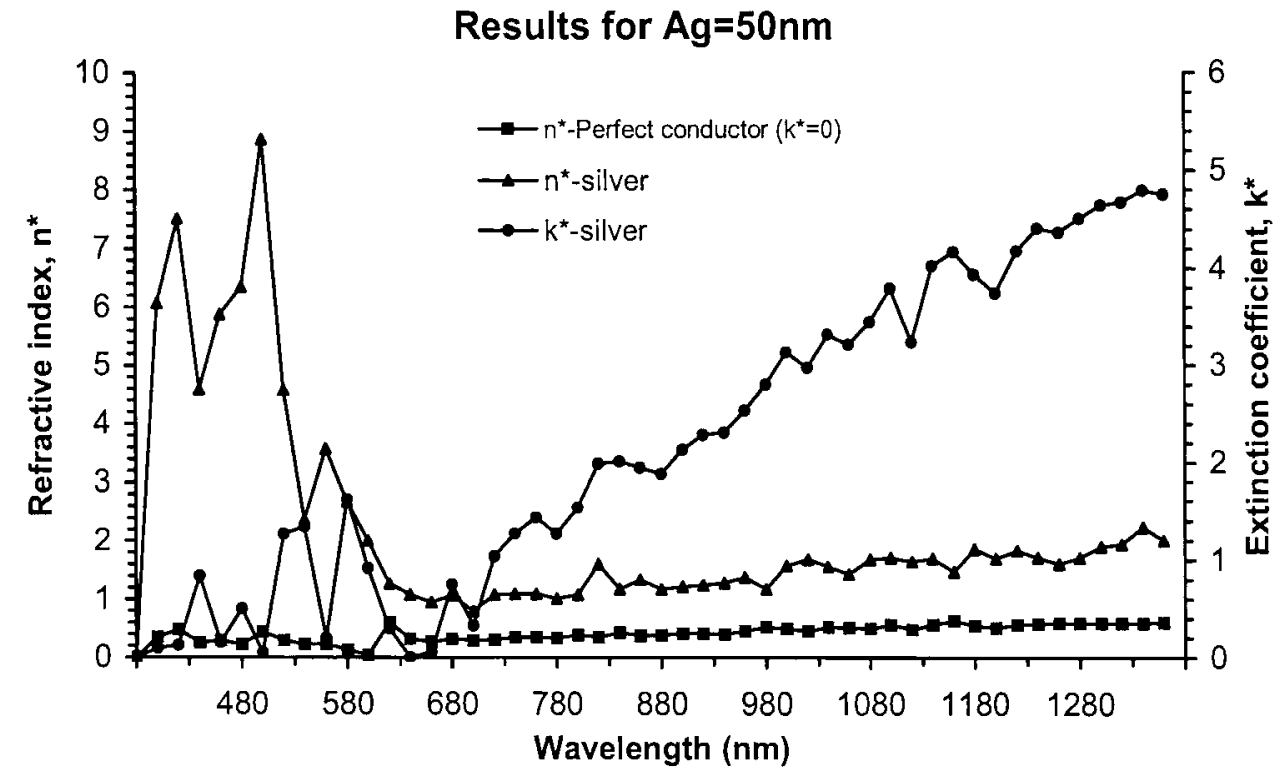

Figure 5(c) Spectral $\mathrm{n}^{*}$ and $\mathrm{k}^{*}$ values for an array of slits in $50 \mathrm{~nm}$ thick layers of perfect conductor and silver

From the insights gained in the simulation studies of the Poynting vector, we link falls in $\mathrm{k}^{*}$ to enhanced transmittance which comes about from channelling energy through the voids. It is surprising that in the tortuous mesoporous network this channelling seems to be even more efficient than that in the very thin layers, but may be linked to the large internal 
surface area of metal. A fractal indirect network of voids spanning a metal layer from one side to the other may be an interesting related example. It may be mathematically tractable, and hence provide insights into the mesoporous system.

These enhancements in $\mathrm{n}^{*}$ do not emerge from classical quasistatic models for our geometries. The strong dependence of $\mathrm{n}^{*}$ on the neighbouring dielectric properties, as shown in figures 4 (a) and (b) when we overcoat the $7 \mathrm{~nm}$ film with oxide and compare $n^{*}$ in that case, with $n^{*}$ of the same film in air, also demands new approaches. $k^{*}$ is much less sensitive to coating than $n^{*}$. Coupled BSPs depend on the media on both sides as well as on surface nanostructure and void percolation. The resonant effects associated with BSPs thus provide some insights. An elevated $n^{*}$ implies that the energy in effect crosses the medium relatively slowly. The coupled BSP state is resonant or partially resonant with incident radiation. The photons can be captured by this state that forms via the cavities ${ }^{14}$ leading to significant effective transit delays, but also high transit probabilities. Damping within the states (in the surface plasmons and their coupling "oscillator" mechanism) must provide the limitation on transmittance efficiency. Otherwise even weak coupling would allow $100 \%$ resonant $\mathrm{T}$ values as in our lossless metal simulations. These states are in effect a virtual bound state for temporary capture of incident photons. Resonant delays for pulses have been observed ${ }^{23}$, and also occur in other conducting metal meso-systems. Surface resonant delays are also linked to achieving physically realisable effective negative refractive indices ${ }^{24}$.

Comparing the $\mathrm{n}^{*}, \mathrm{k}^{*}$ for the ordered array of voids in a $50 \mathrm{~nm}$ thick silver layer (Fig. 5(c)) with those on the random voids in 7 and $10 \mathrm{~nm}$ thick granular silver (Fig. 4(b) we observe similar behaviour . $\mathrm{k}^{*}$ is almost identical in magnitude and spectral behaviour. $\mathrm{n}^{*}$ has a flat spectral response in $7 \mathrm{~nm}$ films and also the simulated case beyond $800 \mathrm{~nm}$. The simulated $n^{*}$ value is well above bulk silver $n$ value, but not as high as that in the granular silver which is also a thinner film. However from the models we have discussed it can be shown that one expects $\mathrm{n}^{*}$ to increase as such layers get thinner and the measured data is for films around $10 \mathrm{~nm}$ compared to the simulated case of $50 \mathrm{~nm}$. Simulated $\mathrm{n}^{*}, \mathrm{k}^{*}$ comparisons between $50 \mathrm{~nm}$ and $500 \mathrm{~nm}$ layers indicate that strong NIR resonant transmittance peaks coupled with very low effective $\mathrm{k}^{*}$ may be a feature of films with thicknesses comparable or larger than wavelength. Away from the peaks $\mathrm{n}^{*}$ is not strongly different from the bulk metal Quite thin layers with voids, are anomalous in a different way. They do not show these distinct NIR resonant peaks but have elevated $n^{*}>1$ across a very broad band. Note that $n^{*}$ for the perfect conductor is qualitatively similar to silver in both thick and thin cases, with $\mathrm{n}^{*}$ small but almost constant.

The lack of sharp resonant peaks in all experimental data and in the $50 \mathrm{~nm}$ thick simulations is noticeable. Only in the thick layer simulations does a clear resonant peak emerge, and this is linked to the establishment of standing waves in the cavity. It thus appears that in both thin and mesoporous layers resonances are broad. In terms of $\Delta_{\mathrm{SP}}$ of equations (4) and (5) which links finally to zeros in the real part of the denominator of equation (6), either (or both) the nanostructured surface features are sufficiently irregular to allow many SP modes to form, or as layers get thinner equation (6) predicts a greater shift in the $\mathrm{k}_{\mathrm{x}}$ dispersion curves from bulk values. These changes with thinning are known to lead to an increasing broadness in the transmittance enhancement factor in regular silver when surface plasmons are activated using prism coupling ${ }^{13}$ and will also occur here since the dependence of $\mathrm{T}$ on thickness is mathematically similar.

\section{CONCLUSION}

Insulating layers containing dense arrays of metal nanoparticles can be modelled with simple effective medium models provided the particles remain randomly distributed and separate. Once macroscopic conduction occurs surface currents, in particular surface plasmons, occur when surface nanostructure is present, and must be accounted for in optical homogenisation models (which assume smooth surface layers). When SP effects are present there is usually a significant enhancement of the effective real part of the index $n^{*}$, and a reduction of the extinction part $\mathrm{k}^{*}$. The reduction in $\mathrm{k}^{*}$ is linked to the enhanced transmittance that occurs when coupling of SPs occurs across thin layers. When SP states are present $n^{*}$ and $\mathrm{k}^{*}$ are sensitive to film thickness, and to the dielectric properties of the adjacent medium. Use of a graded index layer, say with MG or Bruggemann theory to model the "outer layers" for the optical response of a nanostructured metal film will give different results to those from extended surface plasmons.

\section{ACKNOWLEDGEMENTS}

Geoff McCredie has assisted with many thin film preparations, and Ric Wuhrer with high resolution SEM studies. 


\section{REFERENCES}

1. T.G. Mackay, Homogenisation of linear and non linear complex composite materials, Introduction to complex mediums for optics and electromagnetics, (W.S. Weiglhofer and A Lakhtakia eds), SPIE, Bellingham, WA, USA, 2003.

2. G.B. Smith, Nanostructured thin films, Introduction to complex mediums for optics and electromagnetics, (W.S. Weiglhofer and A Lakhtakia eds), SPIE, Bellingham, WA, USA, 2003.

3. G. Zajac, G.B. Smith and A Ignatiev, "Refinement of solar absorbing black chrome microstructure and its relationship to optical degradation mechanisms", J.Appl.Phys., 51, 5544-5554, 1980.

4. Q.C. Zhang ad D.R. Mills, "New cermet films structures with much improved selectivity for solar thermal applications", Appl. Phys. Lett, 60, 545-547, 1992.

5. G.B. Smith, S.Dligatch, R.Sullivan and M.G. Hutchins, "Thin film angular selective glazing", Solar Energy, 62, $229-244,1998$.

6. F. J. Jahan and G.B. Smith, "Investigation of angular selective optical properties of silver/titanium oxide cermet thin films", Thin Solid Films, 333, 185 - 190, 1998.

7. L Ward, in Optical constants of bulk materials and films, Chapter 8, Adam Hilger, Bristol, 1988.

8. G. B. Smith "Dielectric constants for mixed media", J.Phys.D. :Appl Phys ., 10 L39 L42, 1977.

9. T. Ung, L.M. Liz-Marzan and P. Mulvaney, "Optical properties of thin films of $\triangle \mathrm{u}(\hat{a}$ SiO2 particles", J. Phys. Chem. B, 105, 3441-3452, 2001.

10. B. Raguse, J.Herrmann, G. Stevens, J. Myers, G. Baxter, K-H. Muller, T. Reda, A. Molydok and V. BraachMaksvytis, J. Nanopart. Res., 4, 137 - , 2002.

11. L.Harris, R.T. McGuinnes and B.M. Siegel, "The preparation and optical properties of gold blacks", J Opt. Soc Am., 38, 582-589, 1948.

12. A.V. Radchik, A.V. Paley, G.B. Smith and A.V. Vagov, "Polarisation and resonant absorption in intersecting cylinders and spheres", J Appl. Phys. 76, 4827 - 4835, 1994.

13. H.Raether, Surface Plasmons on Smooth and Rough Surfaces and on Gratings, Springer Tracts in Modern Physics 111, Berlin, 1988.

14. L.Martin-Moreno, F.J. Garcia-Vidal, H.J. Lezec, K.M. Pellerin, T.Thio, J.B. Pendry and T.W. Ebbeson, "Theory of exaordinary optical transmissin through subwavelngth hole arrays", Phys. Rev. Lett., 86, 1114-1117, 2001 .

15. T.W. Ebbeson, H.J.Lezec H.F. Ghaemi T.Thio and P.A Wolff, "Extraordinary optical transmission through sub wavelength hole arrays", Nature, 391, 667-669, 1998.

16. R.Dragila and S. Vukovic, "Longitudinal-optical-vibration-induced high transparency of nominally opaque thin films", Phys. Rev. B, 41, 3348-3352, 1990.

17. Dragila and S. Vukovic, "Surface wave-induced high transparency of an overdense warm plasma", Optics Letters, 12, 573-575, 1987.

18. V.A Shubin, A. K. Sarychev, J.P.Clerc and V.M. Shalaev, "Local electric and mangetic fields in semicontinuous metal films: Beyond the quasistatic approximation", Phys Rev. B, 62, 11230-11264, 2000.

19. S. Schelm and G.B. Smith, G. Wei, A.Vella, L.Wiecorek, K.H.Muller and B.Raguse, "Double effective medium model for the optical properties of self assembled gold nanoparticle films cross-linked with di-thiols", Nano Letters 4 (2), 335-339, 2004.

20. G. B. Smith, G.A. Niklasson, J.S.E.M.Svensson and C.G. Granqvist, "Noble -metal- based transparent infra-red reflectors:Experiments and Theoretical analyses for very thin gold films ,_J Appl. Phys., 59, 571-581,1986.

21. J. Bravo-Adad, L.Martin-Moreno and F.J. Gracia-Vidal, "Transmission properties of a single metallic slit: From the sub-wavelength regime to the geometric optics limit", Phys. Rev. B, 69, 026601 1-6, 2004.

22. G. B. Smith, "The scope of effective medium theory for fine metal particle solar absorbers", Appl. Phys. Lett. 35, 668-670, 1979.

23. A.Dogiaru, T. Thio, L.J. Wang, T.W.Ebbeson and H.J. Lezec, "Delay in light transmission through small apertures", Optics letters, 26, 450-452, 2001.

24. S. Foteinopoulou, E.N. Economou and C.M. Soukoulis, "Refraction in media with a negative refractive index", Phys. Rev. Lett. , 90, 107402-1-5, 2003. 